\title{
Platelet Transfusion Refractoriness among Patients with Hematological Malignancies in a Tertiary Center in Saudi Arabia
}

Khadega A Abuelgasim ${ }^{1,2 *}$, Salwa R AIR ashed AlHumaid ${ }^{2,3}$, Atheer S AlOtaibi ${ }^{2,3}$, Munira A AlKhashan ${ }^{2,3}$ and Yosra Z Ali ${ }^{1}$

${ }^{1}$ Department of Oncology, King Abdulaziz Medical City, Riyadh, Saudi Arabia

${ }^{2}$ King Abdullah International Medical Research Center, Saudi Arabia

${ }^{3}$ College of Medicine, King Saud bin Abdulaziz University for Health Sciences, Saudi Arabia

\begin{abstract}
Patients with hematological malignancies (HMs) and that undergoing hematopoietic stem cell transplantation (HSCT) for HMs or other disorders require multiple blood product transfusions. Approximately $30 \%$ of these patients develop platelet transfusion refractoriness (PTR), which increases the risk of serious and potentially fatal bleeding complications. The incidence of PTR in the Saudi population is unknown.

To determine the occurrence of PTR in adult HMs and HSCT patients at King Abdul-Aziz Medical City (KAMC), Riyadh, we performed a study of $29 \mathrm{HMs}$ and HSCT patients. Platelet counts were determined prior to and one hour after each platelet transfusion event. Four (13.8\%) patients qualified for PTR, as they had corrected count increment of less than 5000 following two consecutive platelet transfusions. PTR was detected following 17 of the $102(16.7 \%)$ platelet transfusion events. Subgroup analysis showed a significant association between the patient's diagnosis and the development of PTR (75.0\% CML, 18.9\% NHL, 16.7\% AML, 7.1\% ALL, 0.0\% HL p=0.011). No significant correlations between the development of PTR and either age, history of HSCT or previous exposure to blood products were found. Our observed incidence of PTR in Saudi HM and HSCT patients is significantly lower than the reported worldwide incidence $(24-34 \%)$, which might be attributable to consanguinity within the community. Patients with AML and NHL might be at a higher risk of developing PTR.
\end{abstract}

Keywords: Hematological malignancies; Hematopoietic stem cell transplantation; Platelet transfusion refractoriness

\section{Introduction}

Patients with hematological malignancies (HMs) and those undergoing hematopoietic stem cell transplantation (HSCT) often receive high-dose chemotherapy and are therefore at risk of developing severe thrombocytopenia, requiring long term transfusion support. Many of these patients (18-34\%) develop platelet transfusion refractoriness (PTR) [1-3].

PTR increases the demand for platelet concentrates which are not always readily available by hemotherapy services. PTR increases the risk of transfusion related complications. It also increases the risk of serious and potentially fatal bleeding complications in patients with HMs, while their cancers are in complete remission [4,5]. Despite all these significant consequences, this medical problem is often overlooked, and updated management guidelines are not available [6,7].

Data about PTR incidence in the Middle East region is missing. Using patients derived from the King Abdulaziz Medical City (KAMC) in Riyadh, the objective of this study was to determine the incidence of PTR in patients with HM or those undergoing HSCT, and to identify associated demographic and clinical factors.

\section{Materials and Methods}

\section{Patient demographics and clinical setting}

This is a cross-sectional study conducted in from January to December 2015 at KAMC, Riyadh. After obtaining the approval of the King Abdullah International Medical Research Center Institutional Review Board, a cross-sectional study was conducted in the period from January to December 2015 in KAMC, Riyadh. All patients over 18 years of age with a confirmed HMs diagnosis including Acute Myeloid Leukemia (AML), Acute Lymphoblastic Leukemia (ALL),
Hodgkin's Lymphoma (HL), Non-Hodgkin's Lymphoma (NHL) and Multiple Myeloma (MM) who were admitted to the hematology ward of the KAMC were invited to participate. Patients undergoing HSCT for HMs or other diagnoses, such as hemoglobinopathy and solid tumors, were also invited to participate. We excluded febrile patients and those known to have on-going sepsis, Disseminated Intravascular Coagulopathy (DIC), Venoocclusive Disease (VOD) or Graft Versus Host Disease (GVHD) and those with splenomegaly. All participants were consented for the study after local IRB approval.

\section{Platelet preparation}

Indications for platelet transfusion were a platelet count of less than $15 \times 10^{9} / \mathrm{L}$, active bleeding, and prior to invasive procedures such as central line insertion or removal. Platelet pools were generally composed of 6 units of platelet concentrate. They were either buffy coat-derived platelets pools or apheresis platelets (from a single donor) collected using the Gambro Trima ${ }^{\text {rax }}$ ABC Collection System (Lakewood, Colorado, US). Both products were processed to yield minimums of $3.0 \times 10^{11}$ platelets ( $\geq 6$ random platelet concentrates) and residual leukocyte counts of $\leq 5 \times 10^{6} /$ platelet concentrate. The platelet pools are gamma irradiated using acesium-135 irradiator that delivered a central dose of $2500 \mathrm{cGy}$. In all cases, transfused platelets

*Corresponding author: Khadega A Abuelgasim, Department of Oncology, King Abdulaziz Medical City, Riyadh, Saudi Arabia, Tel: 18011111; Fax: 9668011111; E-mail: ahmedkh1@ngha.med.sa

Received October 25, 2016; Accepted November 11, 2016; Published November 18,2016

Citation: Abuelgasim KA, AlHumaid SRA, AlOtaibi AS, AlKhashan MA, Ali YZ (2016) Platelet Transfusion Refractoriness among Patients with Hematological Malignancies in a Tertiary Center in Saudi Arabia. J Oncol Transl Res 2: 112.

Copyright: ( 2016 Abuelgasim KA, et al. This is an open-access article distributed under the terms of the Creative Commons Attribution License, which permits unrestricted use, distribution, and reproduction in any medium, provided the original author and source are credited. 
Citation: Abuelgasim KA, AlHumaid SRA, AlOtaibi AS, AlKhashan MA, Ali YZ (2016) Platelet Transfusion Refractoriness among Patients with Hematological Malignancies in a Tertiary Center in Saudi Arabia. J Oncol Transl Res 2: 112

were either $\mathrm{ABO}$-identical or compatible with the recipients 'blood group.

\section{Evaluation of the platelet transfusion response}

Blood samples were collected from each patient prior to and one hour after platelet transfusion. The response to transfusion was evaluated by calculating the corrected count increment (CCI) $1 \mathrm{~h}$ after each platelet transfusion as follows $[8,9]$.

$$
\mathrm{CCI}=[(\mathrm{A}-\mathrm{B}) \times \mathrm{BSA}] / \mathrm{C} \times 10^{11}
$$

A: platelet count one hour post transfusion

B: pre-transfusion platelet count

\section{BSA: Body Surface Area}

\section{C: number of transfused platelets}

Patients were considered refractory to platelets when they show a post transfusion platelet CCI value of less than 5000 in two consecutive platelet transfusions.

\section{Statistical Analysis}

Descriptive data are presented as mean \pm standard deviation (SD) for the continuous variables. Categorical variables are presented as the frequencies and percentages. Chi-squared tests were utilized to identify correlations and associations between the variables. Differences exhibiting $\mathrm{p}$ values of $<0.05$ were considered statistically significant.

\section{Results}

A total of 29 patients were recruited; 23 (79.3\%) were males and the age was $37.5 \pm 14.1$ years old (mean \pm SD). The most common diagnosis was AML at $9(31.0 \%)$ followed by NHL at $8(27.6 \%)$ and ALL at $6(20.7 \%)$ (Table 1). Fourteen participants received HSCT. A summary of the participant characteristics are illustrated in Table 1.

During the study period, four patients (13.8\%) were diagnosed with PTR, defined as having CCI of less than 5000 following two consecutive platelet transfusion events. In 102 evaluated platelet transfusion events, PTR was detected in 17 (16.7\%). Subgroup analysis showed significant association between PTR and the patient's diagnosis (75.0\% CML, 18.9\% NHL, 16.7\% AML, 7.1\% ALL, 0.0\% HL), $\mathrm{p}=0.011$. In an adjusted analysis, after controlling for gender as a confounding factor, the association between diagnosis and PTR remains significant ( $p$-value $=0.008$ ). No significant correlations between the development of PTR and either age, history of HSCT or previous exposure to blood products were found (Table 2). Bivariate analysis investigating the relationship between events' characteristics and platelet transfusion refractoriness is illustrated in Table 2.

\section{Discussion}

Despite the widespread use of leukoreduction (the removal of white blood cells) and ultraviolet gamma irradiation of blood products to reduce alloimmunization in HMs and HSCT patients after The Trial to Reduce Alloimmunization to Platelets (TRAP) trial results became available; PTR remains a significant problem occurring in up to $34 \%$ of patients $[1,2,10,11]$.

In this study, we observed a PTR prevalence of $13.8 \%$, which is considered to be relatively lower than the reported international rates. This difference might be explained by the high prevalence of consanguinity in the Saudi community, reaching up to $60 \%$ of all marriages. Since first degree cousins and double first degree cousins marriages are very common in the Saudi community, the inbreeding coefficient, which represents a measure for the proportion of loci at which the offspring is expected to inherit identical gene copies from both parents, can reach up to 0.125 [12]. Due to the genetic homogeneity, Saudi HMs patients have higher chance of having a full HLA matched donor within the family, including parents and off springs and possibly within the blood donor community [13]. Transfusing HLA matched platelets, unintentionally, may have contributed to the lower incidence of PTR in our study. Consanguinity may also lead to less variability in the minor histocompatibilty genes known to elicit immune response and therefore decrease the incidence of alloimmunization, hence less PTR [14].

Another factor that might contribute toward the low incidence of PRT at the KAMC is the way platelets are treated prior to transfusion. All blood products that are transfused to HM and HSCT patients undergo leukoreduction and ultraviolet irradiation. Furthermore, most of the patients enrolled on our study received $\mathrm{ABO}$ matched apheresis/ single donor platelets, which proven to reduces PTR rates $[1,11]$. Although in TRAP study there was no additional benefit in apheresis compared to pooled platelets [10]. We did not look specifically at the difference in CCIs among recipients of the 2 different platelet products.

\begin{tabular}{|c|c|}
\hline Characteristics & N (\%) \\
\hline Gender & \\
Male & $23(79.3)$ \\
Female & $6(20.7)$ \\
\hline Diagnosis & $9(31)$ \\
AML & $6(20.7)$ \\
ALL & $3(10.3)$ \\
HL & $8(27.6)$ \\
NHL & $1(3.4)$ \\
CML & $2(6.9)$ \\
Others & $14(48.3)$ \\
\hline HSCT & $15(51.7)$ \\
Yes & $29.6 \pm 22.2$ \\
\hline No & \\
\hline Number of prior transfusions - mean \pm SD & \\
\hline
\end{tabular}

Table 1: Demographic and clinical characteristics of participants.

ALL: Acute Lymphoid Leukemia; AML: Acute Myeloid Leukemia; CML: Chronic Myeloid Leukemia; Hodgkin's Lymphoma (HL), HSCT: Hematopoietic Stem Cell Transplantation; NHL: Non-Hodgkin's Lymphoma; Others: Testicular Cancer and Multiple Myeloma

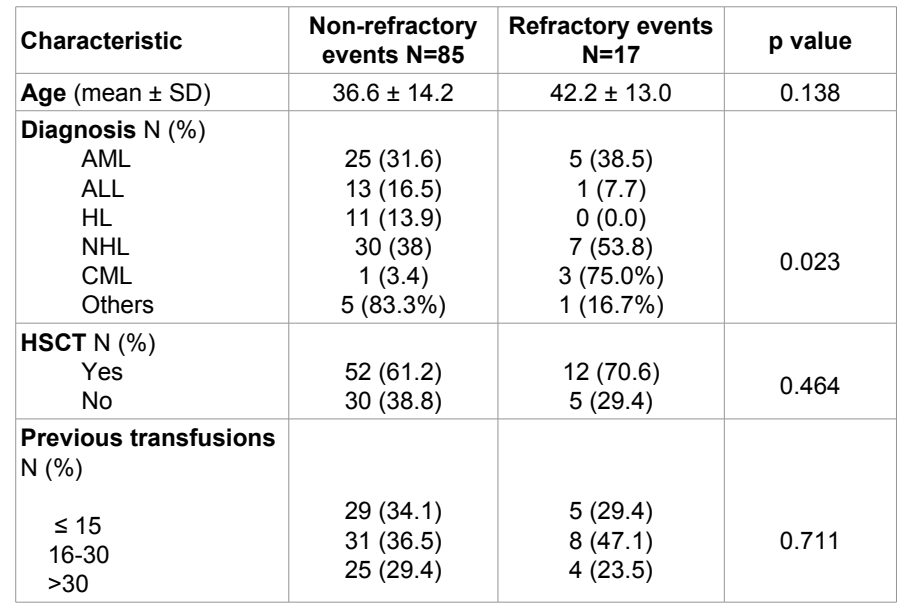

Table 2: Bivariate analysis investigating the relationship between events characteristics and platelet transfusion refractoriness.

AML: Acute Myeloid Leukemia; ALL: Acute Lymphoid Leukemia; CML: Chronic Myeloid Leukemia; HL: Hodgkin's Lymphoma; HSCT: Hematopoietic Stem Cell Transplantation; NHL: Non-Hodgkin's Lymphoma; Other: Testicular Cancer and Multiple Myeloma. 
Citation: Abuelgasim KA, AlHumaid SRA, AlOtaibi AS, AlKhashan MA, Ali YZ (2016) Platelet Transfusion Refractoriness among Patients with Hematological Malignancies in a Tertiary Center in Saudi Arabia. J Oncol Transl Res 2: 112

We recommend studying the difference in PTR incidence when pooled vs. apheresis platelets are used in our patients' population in the future.

The presence of antibodies against human platelet antigen (HPA) or against human leukocyte antigen (HLA) class I (PRA-HLA) due to prior exposure from pregnancy, blood products or stem cells transfusion, indicate alloimmunization and is associated with the development of PTR [7,11,13-16]. Anti-HPA antibodies are present in one-third of HM patients who developed PTR after receiving multiple blood product transfusions [15,17]. Anti-HLA antibodies are more common, occurring in up to $67 \%$ of refractory patients. Anti-HLA antibodies are thought to be the primary cause for immunological PTR $[14,16,18]$. Anti-HPA antibodies are more common in patients who have Anti HLA antibodies secondary to multiple transfusion exposures compared to those who PRA-HLA negative $[17,19]$.

The combined use of HLA- and ABO-matched platelets, rather than $\mathrm{ABO}$-matched platelet concentrates alone, is usually recommended for the management of PTR patients who test positive for anti-HLA and anti-HPA antibodies [7]. However, the use of HLA-matched platelets in HM patients with PTR is not always feasible in many tertiary centers worldwide. Moreover, HLA-matched platelets in allogeneic HSCT candidates should be avoided due to the increased risk of graft rejection [18-21].

In our study, we did not test for the presence of either PRA-HLA or anti-HPA antibodies. This is however to be planned as part of a future multicenter study in Saudi Arabia. If the association between PRA-HLA positivity and PTR is proven in our patients' population, recommending using HLA matched platelet transfusion in refractory patients will be appropriate provided that the patient is not a candidate for HSCT.

Non-immunological PTR causes include sepsis, DIC, VOD, Graft versus host disease GVHD, splenomegaly and medications [11,20-23]. In our study, we excluded thrombocytopenic patients with established DIC, VOD, GVHD or splenomgaly. We did not exclude participants only based on their medication history.

Our data did not indicate significant association between PTR and the patients' age or prior history of HSCT. Unexpectedly, we also did not find a significant association between PTR and the prior exposure to blood products, which we find hard to explain.

We found clear associations between diagnoses of AML and NHL and the development of PTR. Similar results have previously been reported by Ferreira et al. [1]. We believe that the small sample size in both of our studies (16 patients in Ferreira et al. [1]) is a limiting factor; however, we were able to replicate their findings. In Ferreira et al. a higher incidence of PTR was detected among females, particularly in those with prior pregnancies [1]. In Slichter et al. PTR was more common in males, however, 2 or more pregnancies was associated with higher incidence of PTR in females [11]. We were unable to test these correlations due to the small number of female patients enrolled in our study; however this can be further explored in a future multi-center study.

It is interesting that PTR is more common in AML and NHL patients compared to other HMs such as ALL. Although ALL patients require high dose chemotherapy leading to severe suppression of hematopoiesis necessitating multiple transfusions of blood products, PTR is less common in those patients. This suggests a possible role for different epigenetic mechanisms resulting in more alloimmunzation in AML and NHL patients.
Emerging evidence indicates that epigenetic modifiers might serve as the promising therapeutic targets for the treatment of cancers. For instance, in patients with $\mathrm{HMs}$, inhibition of histone methyltransferase G9a has been found to facilitate the treatment of AML [24]. As the main epigenetic roles of $\mathrm{G} 9 \mathrm{a}$ is depositing of $\mathrm{H} 3 \mathrm{~K} 9 \mathrm{me} 2$ at euchromatin and maintenance of DNA methylation at imprinted control regions $[25,26]$. Aberrant distribution of G9a-associated epigenetic markers might be the causes of HMs in humans. Apart from histone methyltransferase, mutations within DNA methyltransferase 3A (DNMT3A) gene is also strongly associated with poor prognosis in de novo AML patients [27]. Therefore, abnormal gain or loss of DNA methylation might also lead to the initiation and development of AML and mutations within DNMT3A may be a potential biomarker for the diagnosis of this disease.

Our data did not indicate significant association between PTR and the patients' age or prior history of HSCT. Unexpectedly, we also did not find a significant association between PTR and the prior exposure to blood products, which we find hard to explain.

To manage HMs patients with PTR, multiple strategies are used including: transfusing bigger dosed of platelets, using steroids, using intravenous immunoglobulin or anti-D in patients who are Rh-positive and using $\mathrm{ABO}$ matched platelets concentrates. Using HLA A and $\mathrm{B}$ antigen matched platelets concentrates is the mainstay of managing such patients, however it leads to satisfactory increments in only half of alloimmunzed patients [28]. Platelet cross-matching is a simple and rapid procedure that can be used to select suitable donor platelets for alloimmunized patients $[29,30]$.

\section{Conclusion and Recommendations}

Given the significant incidence of PTR in Saudi Arabia, although lower than international reports, the implementation of preventative and therapeutic measures for the management of PTR in HMs and HSCT patients must remain a focus. We recommend that larger, multicenter studies be conducted to further probe the incidence of PTR, and to analyze the correlations of PTR with demographic and clinical factors, different blood products and also to evaluate the presence of PRA-HLA and anti-HPA antibodies in patients who develop PTR. We also recommend investigating the potential benefit of HLA-matched platelets in the management of patients who qualify for PTR.

\section{Declaration of Interest}

This study was not funded and the authors declare having no conflict of interest.

\section{Disclosure}

All authors contributed in generating the research idea, designing the study protocol, collecting/interpreting data and writing research proposal and manuscript. All authors approved the final manuscript.

\section{References}

1. Ferreira AA, Zulli R, Soares S, Castro Vd, Moraes-Souza H (2011) Identification of platelet refractoriness in oncohematologic patients. Clinics (Sao Paulo) 66 $35-40$

2. Li G, Liu F, Mao X, Hu L (2011) The investigation of platelet transfusion refractory in 69 malignant patients undergoing hematopoietic stem cell transplantation. Transfus Apher Sci 45: 21-24.

3. Slichter SJ (1997) Algorithm for managing the platelet refractory patient. J Clin Aph 12: 4-9.

4. Kerkhoffs JL, Eikenboom JC, van de Watering LM, van Wordragen-Vlaswinke 
Citation: Abuelgasim KA, AlHumaid SRA, AlOtaibi AS, AlKhashan MA, Ali YZ (2016) Platelet Transfusion Refractoriness among Patients with Hematological Malignancies in a Tertiary Center in Saudi Arabia. J Oncol Transl Res 2: 112.

RJ, Wijermans PW, et al. (2008) The clinical impact of platelet refractoriness: Correlation with bleeding and survival. Transfusion 48:1959-1965.

5. Toor AA, Choo SY, Little JA (2000) Bleeding risk and platelet transfusion refractoriness in patients with acute myelogenous leukemia who undergo autologous stem cell transplantation. BMT 26: 315-320.

6. Quaglietta A, Nicolucci A, Accorsi P, Pompa A, Pierelli L, et al. (2012) Survey of current practice for monitoring and management of platelet refractoriness in Italy. Transfus Apher Sci 47: 271-276.

7. Novotny VM (1999) Prevention and management of platelet transfusion refractoriness. Vox Sang 76: 1-13.

8. Daly PA, Schiffer CA, Aisner J, Wiernik PH (1980) Platelet transfusion therapy. One hour post-transfusion increments are valuable at predicting the need for HLA-matched preparation. JAMA 243: 435-438.

9. Herman J (2005) Platelet transfusion therapy. In Mintz PD (ed): Transfusion therapy: Clinical principles and practices. Bethesda. MD. AAB Press.

10. Leukocyte reduction and ultraviolet $B$ irradiation of platelets to prevent alloimmunization and refractoriness to platelets transfusion (1997) The trial to reduce alloimmunization to platelets study group. N Eng J Med 337: 18611869.

11. Slichter SJ, Davis K, Enright H, Braine H, Gernsheimer T, et al. (2005) Factors affecting posttransfusion platelet increments, platelet refractoriness and platelet transfusion intervals in thrombocytopenic patients. Blood 105: 4106-4114.

12. El-Hazmi MA, al-Swailem AR, Warsy AS, al-Swailem AM, Sulaimani R, et al. (1995) Consanginity among the Saudo Arabian Population. J Med Genet 32: 623-626.

13. Hamamy H (2012) Consanguineous marriages. Preconception consultation in primary health care settings. J Community Genet 3: 185-192.

14. Hajeer AH, Alqattan M, Anizi A, Alaskar AS, Jarrar MS (2012). Chances of finding a matched parent-child in hematopoietic stem cell transplantation in Saudi Arabia. Am J Blood Res 2: 201-202.

15. Mehra NK (2001) Histocompatibilty Antigens. eLS.

16. Delaflor-Weiss E, Mintz PD (2000) The evaluation and management of platelet refractoriness and alloimmunization. Transfus Med Rev 14:180-196.

17. Fabris F, Soini B, Sartori R, Randi ML, Luzzatto G, et al. (2000) Clinical and laboratory factors that affect the post-transfusion platelet increment. Transfus Sci 23: 63-68.

18. Agarwal N, Chatterjee K, Sen A, Kumar P (2014) Prevalence of platelet reactive antibodies in patient's refractory to platelet transfusion. Asian J Transfus Sci 8 126-127.

19. Laundy GJ, Bradly BA, Rees BM, Younie M, Hows JM (2004) Incidence and specificity of HLA antibodies in multitransfused patients with acquired aplastic anemia. Transfusion 44: 814-825.

20. Kickler T, Kennedy SD, Braine HG (1990) Alloimmunization to plateletspecific antigens on glycoprotein Ilb-IIla and Ib/IX in multiply transfused thrombocytopenic patients. Transfusion 30: 622-625.

21. Champlin RE, Horowitz MM, van Bekkum DW, Camitta BM, Elfenbein GE, et al. (1989) Graft failure following bone marrow transplantation for severe aplastic anemia: Risk factors and treatment results. Blood 73: 606-613.

22. Desmarets M, Cadwell CM, Peterson KR, Neades R, Zimring JC (2009) Minor histocombatibility antigens on transfused leukoreduced units of red blood cells induce bone marrow transplant rejection in a mouse model. Blood 114: 2315 2322.

23. Hess JR, Trachtenberg FL, Assmann SF, Triulzi DJ, Kaufman RM, et al. (2016) Clinical and laboratory correlates of platelet alloimmunization and refractoriness in the PLADO trial. Vox Sang.

24. Hod E, Schwartz J (2008) Platelet transfusion refractoriness. Br J Hematol 143 348-360.

25. Lehertz B, Pabst C, Su L, Miller M, Liu F, et al (2014) The methyltransferase G9a regulates HoxA9-dependant transcription in AML. Genes Dev 28: 317327

26. Tachibana M, Suqimoto K, Nozaki M, Ueda J, Ohta T, et al. (2002) G9a histone methyltransferae plays a dominant role in euchromatic histone $\mathrm{H} 3$ lysine 9 methylation and is essential for early embryogenesis. Genes Dev 16: 17791791.

27. Zang T, Termanis A, Ozkan B, Bao XX, Culley J, et al. (2106) G9a/GLP complex maintains imprinted DNA methylation in embryonic stem cells. Cell Rep 15: 77-85.

28. Hou HA, Kuo YY, Liu CY, Chou WC, Lee MC, et al. (2012) DNMT3A mutations in acute myeloid leukemia: Stability during disease evolution and clinical implications. Blood 119: 559-568.

29. Duquensoy RJ, Filip DJ, Rodney GE, Rimm AA, Aster RH (1997) Successful transfusion of platelets 'mismatched' for HLA antigens to alloimmunized thrombocytopenic patients. Am J Hematol 2: 219-226.

30. Sacher RA, Kickler TS, Schiffer CA, Sherman LA, Bracey AW, et al. (2003) Management of patients refractory to platelet transfusion. Archives of Pathology and Laboratory Medicine 127: 409-414. 